

\section{Sistema geotérmico hidrotermal del volcán Azufral}

Claudia María Alfaro Valero

Gina Rodríguez Ospina

Gilbert Fabián Rodríguez Rodríguez

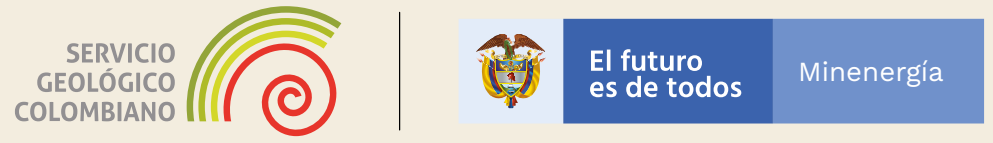


@ Servicio Geológico Colombiano

Oscar Paredes Zapata

Director general

Mario Andrés Cuéllar

Director de Geociencias Básicas

ISBN impreso: 978-958-53131-6-3

ISBN digital: 978-958-53131-7-0

DOI: https://doi.

org/10.32685/9789585313170

\section{(c) (i)}

(C) Servicio Geológico

Colombiano. (2021).

Esta obra es distribuida bajo licencia internacional Creative Commons

Atribución/Reconocimiento 4.0

\section{Autores}

Claudia María Alfaro Valero

Gina Rodríguez Ospina

Gilbert Fabián Rodríguez Rodríguez

\section{Comité Editorial}

Presidente

Juan Guillermo Ramírez

Integrantes

Mario Andrés Cuéllar

Gloria Prieto Rincón

Marta Calvache Velasco

Hernán Olaya Dávila

Humberto Andrés Fuenzalida

Hernando Camargo

Victoria Eugenia Díaz

Virgilio Amaris

Viviana Dionicio

Teresa Duque

Julián Escallón

Armando Espinosa

Constanza Martínez
Diagonal 53 n. ${ }^{\circ}$ 34-53

Bogotá D. C., Colombia

Teléfono: (601) 2200200,

ext.: 3048

ceditorial@sgc.gov.co

Henry Villegas

Corrección de estilo

Diana Paola Abadía

Diseño y diagramación

Carolina Hernández

Editora general

Fotografías de cubierta:

Archlvo SGC

Acuarela: Manuel María Paz, 1853.

Comisión Corográfica

Bogotá, Colombia

Noviembre de 2021

Citación: Alfaro, C., Rodríguez, G.

y Rodríguez-Rodríguez, G. (2021).

Sistema geotérmico hidrotermal

del volcán Azufral. Servicio

Geológico Colombiano. https://doi.

org/10.32685/9789585313170 


\section{Agradecimientos}

El Servicio Geológico Colombiano agradece a las comunidades indígenas y demás residentes de las zonas rurales de los municipios de Túquerres, Mallama, Guachucal, Sapuyes y Guachavés, y a las autoridades ambientales y municipales correspondientes, por autorizar los trabajos de campo que permitieron investigar y generar nuevo conocimiento del área geotérmica del volcán Azufral, información resumida en este documento.

Un agradecimiento muy especial a la doctora Esperanza Agreda Montenegro, miembro de número de la Academia Nariñense de Historia, por su participación en la revisión del borrador del documento. 
Este documento se elaboró para compartir, con las comunidades locales del área geotérmica del volcán Azufral, el conocimiento generado a través de las investigaciones realizadas por el Servicio Geológico Colombiano (SGC), utilizando un lenguaje familiar, cálido y amigable. Esperamos que así sea percibido por las comunidades. El documento, complementa los informes técnicos elaborados en desarrollo de la Investigación geotérmica en Azufral, Nariño. Dado que la información generada por el SGC es de carácter público, se informa que podrá ser descargada por todos los ciudadanos a través del Motor de Integración de Información Geocientífica, de la institución, disponible en internet <https://miig.sgc.gov.co>.

Nuestro sueño, como grupo de tra-

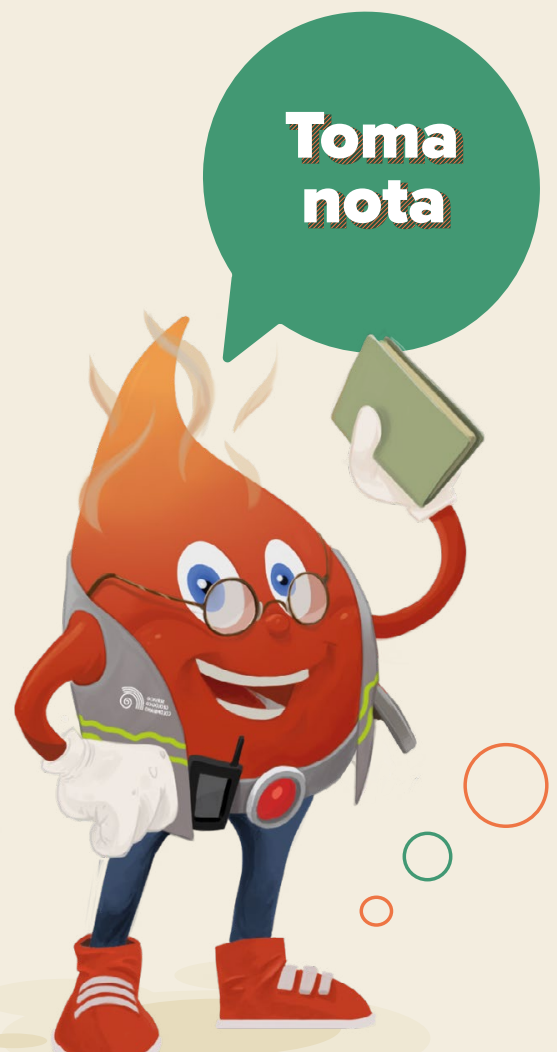
bajo, es integrar este conocimiento generado desde trabajos de investigación científica, con los saberes ancestrales y tradicionales, así como con la visión poética de los mitos y leyendas que forman parte de la riqueza cultural de los indígenas del territorio. Sea esta una invitación para generar espacios de compilación, discusión e integración de estas dos fuentes de conocimiento.

\section{Los integrantes del Grupo} Investigación Geotérmica del SGC les enviamos un afectuoso saludo. 


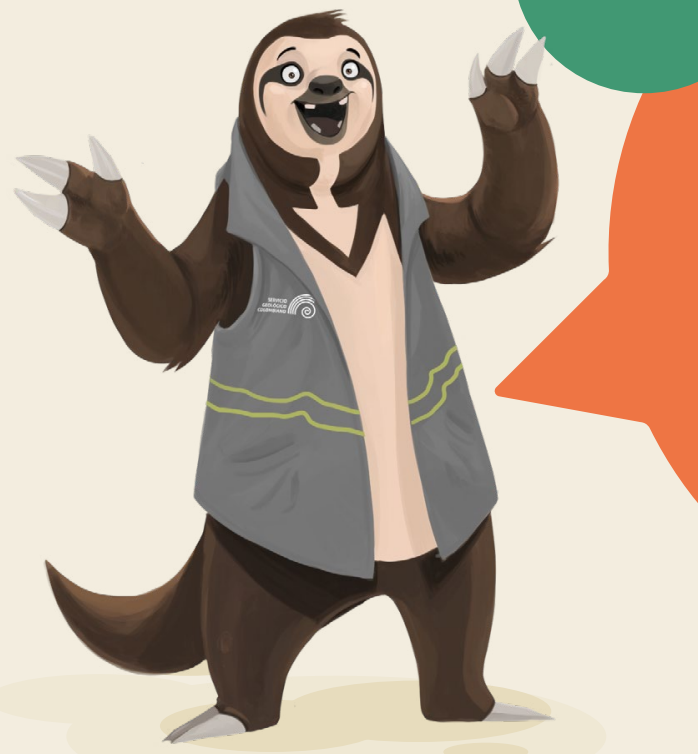

\section{soy Geo,}

la mascota del Servicio Geológico Colombiano. Soy un eremoterio (perezoso gigante); aunque recorrí la Tierra hace millones de años, estoy aquí junto con mi amigo Chitu para contarte cómo funciona el sistema geotérmico hidrotermal del volcán Azufral

o Gran Chaitán.

yo soy una gota de agua y me llamo Chitu, que significa caliente en lengua muisca;

también puedes Ilamarme Achachaq o Kuñi, que tienen el mismo significado, pero en lengua quechua. Tengo mucha energía porque vivo bajo la superficie de la Tierra, donde me caliento y puedo circular a través de las rocas. Durante mis recorridos veo cosas impresionantes y hermosas, que quiero compartir con mi amigo Geo y contigo. 


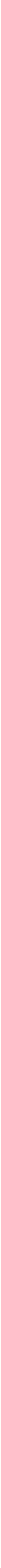




\section{El Gran Chaitán}

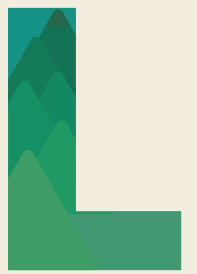

a historia del volcán nace a partir del amor de una pareja anterior a la comunidad de los Pastos. El amor de ellos fue bendecido por los dioses, que les concedieron tener un hijo, que pudo crecer fuerte y sano gracias al cuidado de sus padres. Un día el papá al regresar a casa no encontró a su hijo ni a su esposa; los buscó por todos lados, en todos los caminos posibles; pasaron cerca de 33 lunas pero no aparecieron, como si las feroces bestias de la zona donde vivían se los hubieran comido. Pero esto no fue así, los dioses se los habían llevado a ambos. La razón que le dieron al padre era que su hijo se convertiría en un guerrero dispuesto a luchar, en un soldado valiente que sería conocido como El Chispas, y que algún día pelearía contra un brujo llamado El Guangas. Estos dos brujos poderosos, El Chispas y El Guangas, por su relación conflictiva intentarían definir el origen del cosmos, convirtiéndose en tigres para luchar luego al interior de una cesta. A partir de entonces, se dice que el mundo como lo conocemos quedó todo al revés; lo bueno quedó malo, lo malo bueno, lo de adentro para afuera, lo de afuera para adentro, y así sucesivamente. Una vez se termine este encantamiento todo el mundo volverá a su estado normal.

El padre triste por la soledad, y resignado a la ausencia de sus seres queridos, se sentó en la montaña más alta, y en un último suspiro comenzó a llorar muy fuerte, recordando a su esposa e hijo. Fue tanto el dolor que sintió, y tantas las lágrimas que cayeron sobre la montaña, que se formaron las tres lagunas: la laguna negra por su dolor, la blanca por su resignación y la verde por su esperanza. Los dioses al percatarse del sufrimiento de aquel hombre, lo convirtieron en el volcán, bautizado por ellos como el Gran Chaitán. Es por eso que, desde ese momento y de forma continua, lanza suspiros de dolor, azufre y gas a las tierras bajas. Estos quejidos y lamentos han sido escuchados por el Taita-Urcos (volcán 
Galeras), quien en forma de reproche lanza brotes de ceniza como señal de molestia con el Chaitán, por romper la paz de las entrañas de la tierra. Hoy en día, la magia aún reina en el Gran Chaitán y sus zonas aledañas. Dice la sabiduría ancestral que es por esto que se debe transitar con mucho cuidado y silencio, para evitar despertar el espíritu del Gran Chaitán. Los niños que se acercan a la laguna son los más propensos a perderse porque representan de cierta forma a El Chispas, convirtiéndose en un consuelo de aquel volcán enamorado (modificado de Patiño et al., 2009).

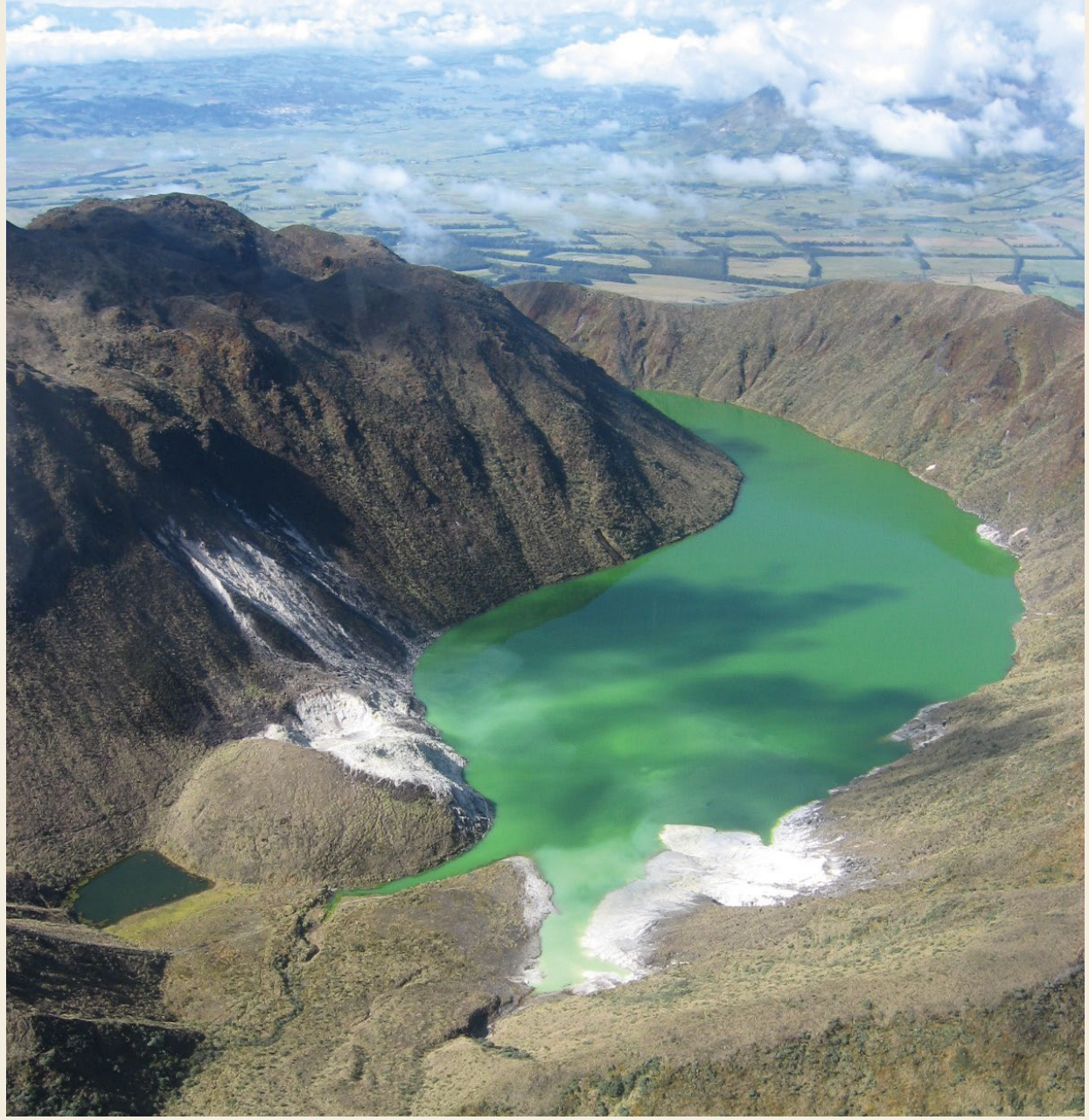

Fuente: SCG (2008) 
Es hermoso el Gran Chaitán, yo lo he recorrido y además de la Laguna Verde, he sentido su suelo caliente y he visto sus fuentes termales en las que me he sumergido a calentarme.

Sí, todo esto hace parte del sistema geotérmico hidrotermal del volcán Azufral.

Es un espacio del subsuelo, en donde rocas calientes y permeables permiten almacenar y calentar el agua que viene de la lluvia.

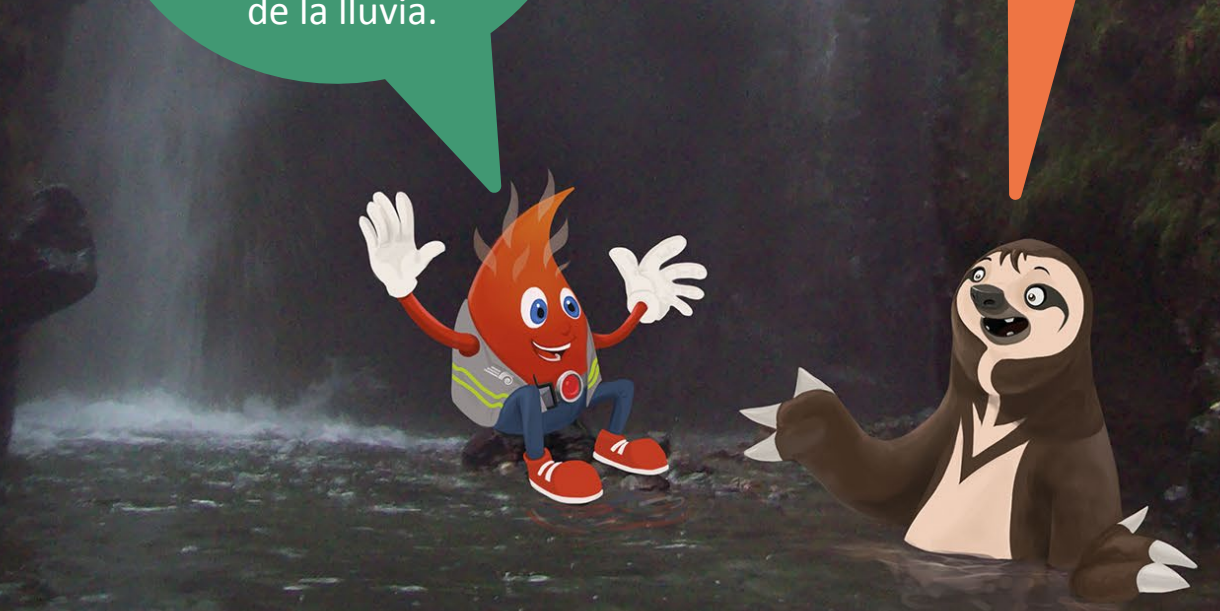




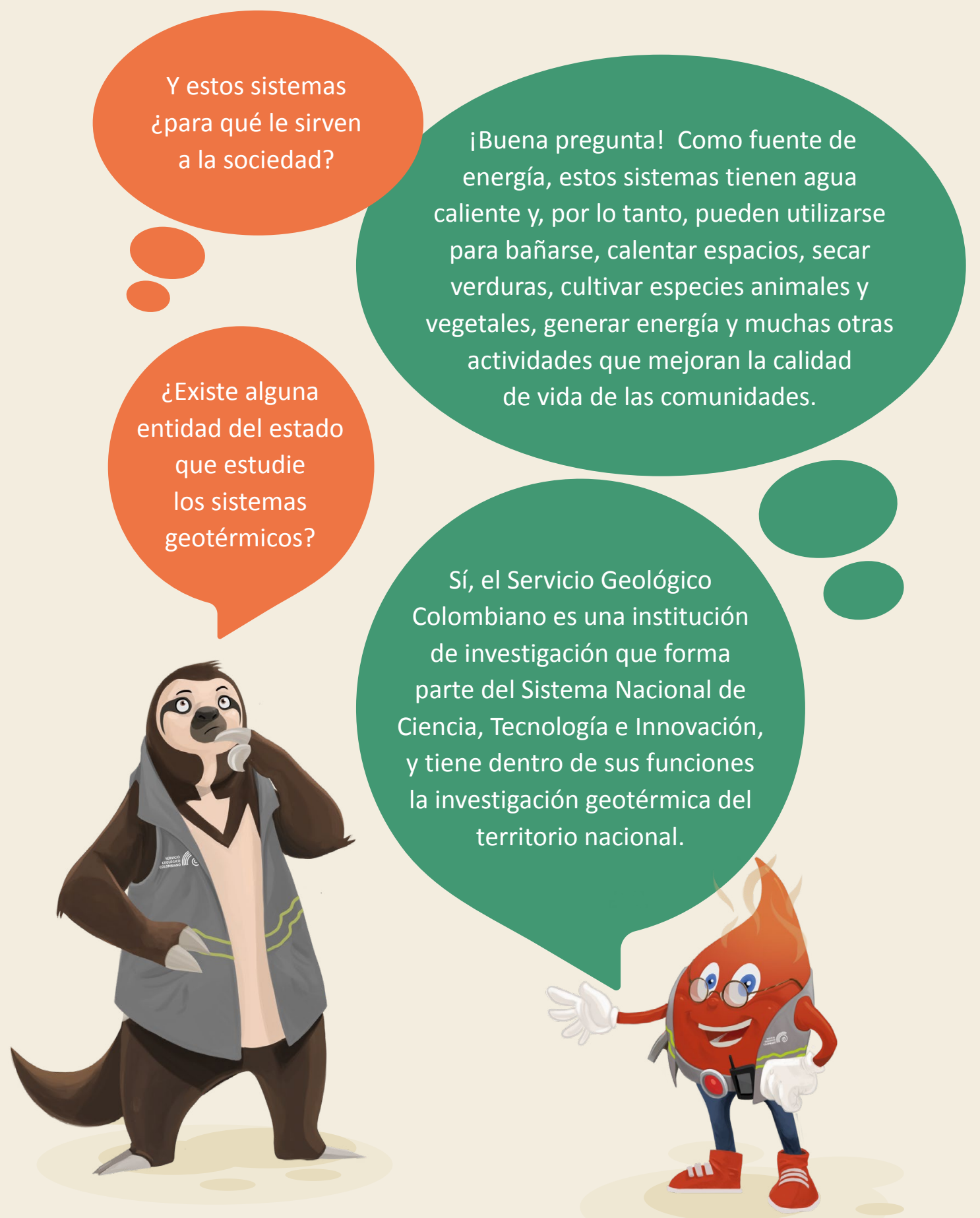


En el volcán Azufral el SGC ha realizado varios estudios, para conocer su sistema geotérmico. Estos estudios incluyen: el mapa geológico, la caracterización de las aguas y gases de los manantiales termales y fumarolas, así como gases contenidos en aire del suelo, estudios geofísicos basados en las mediciones desde la superficie de propiedades magnéticas, eléctricas, gravimétricas y acústicas; variaciones de la temperatura superficial del suelo y la elaboración de modelos geológicos en tres dimensiones, y modelos conceptuales que integran todos los estudios realizados, con el fin de interpretar el sistema geotérmico. Toda la información es generada bajo el criterio de respeto al ambiente, permite a las comunidades conocer sus recursos naturales y puede ser aplicada para planear su protección, y decidir sobre su posible utilización. Puedes solicitar los informes de estos trabajos (cliente@sgc.gov.co) o descargarlos de nuestro Motor de Integración de Información Geocientífica (miig.sgc.gov.co).
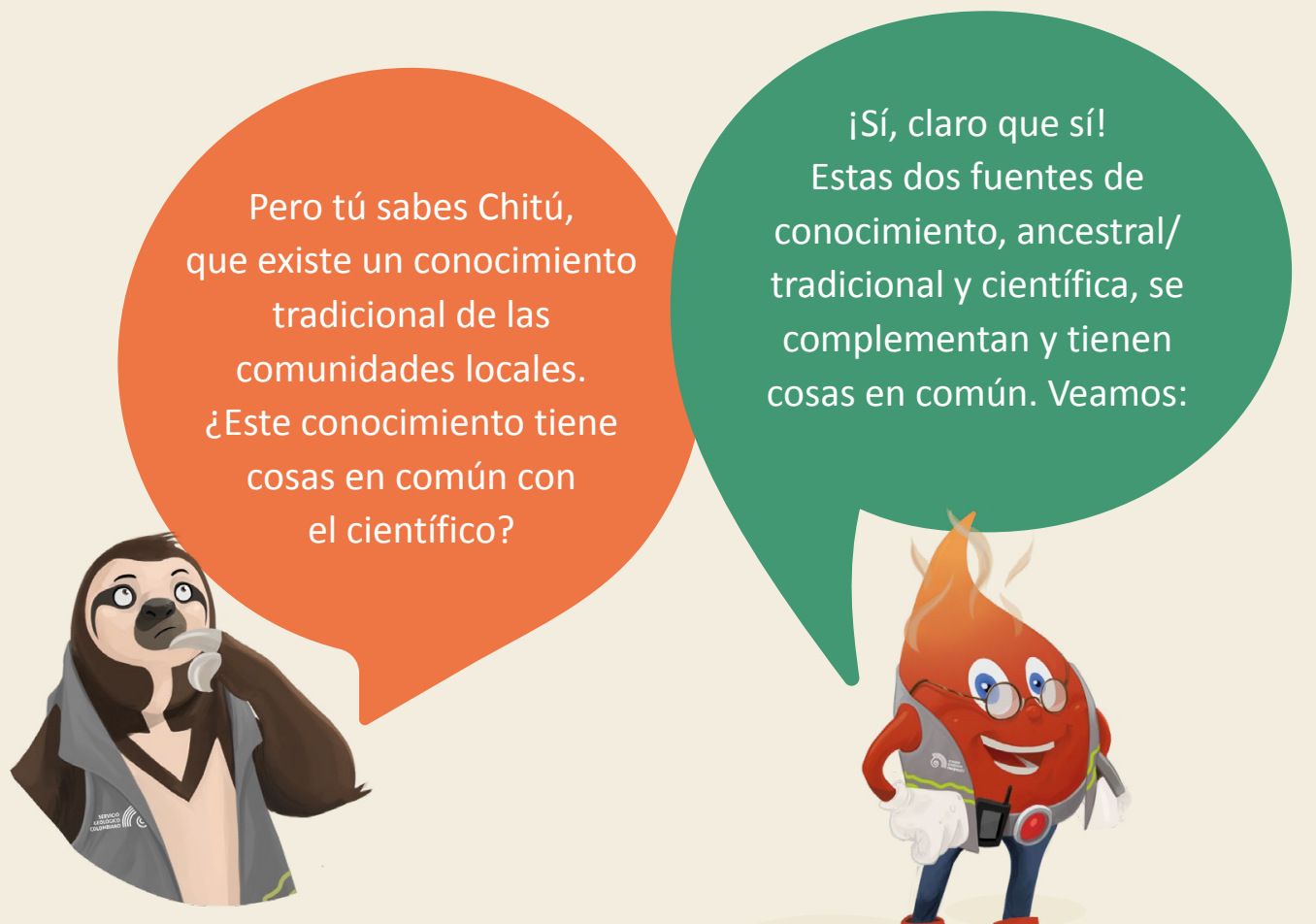


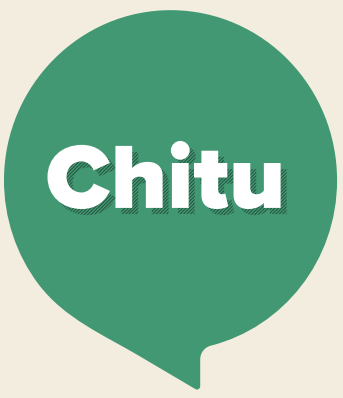

Según los documentos que he leído, el mundo para Los Pastos y otros pueblos andinos se ordena desde una concepción de la verticalidad cósmica, en tres dimensiones representadas en el churo cósmico: el mundo de arriba, el mundo del medio y el mundo de abajo; nombres equivalentes en lengua quechua a Hanan Pacha, Kay Pacha y Uku Pacha, respectivamente. La conformación y representación de mundos es descrita por el pueblo indígena del territorio de Túquerres, de la siguiente forma (Fundación Proyectar, 2009):

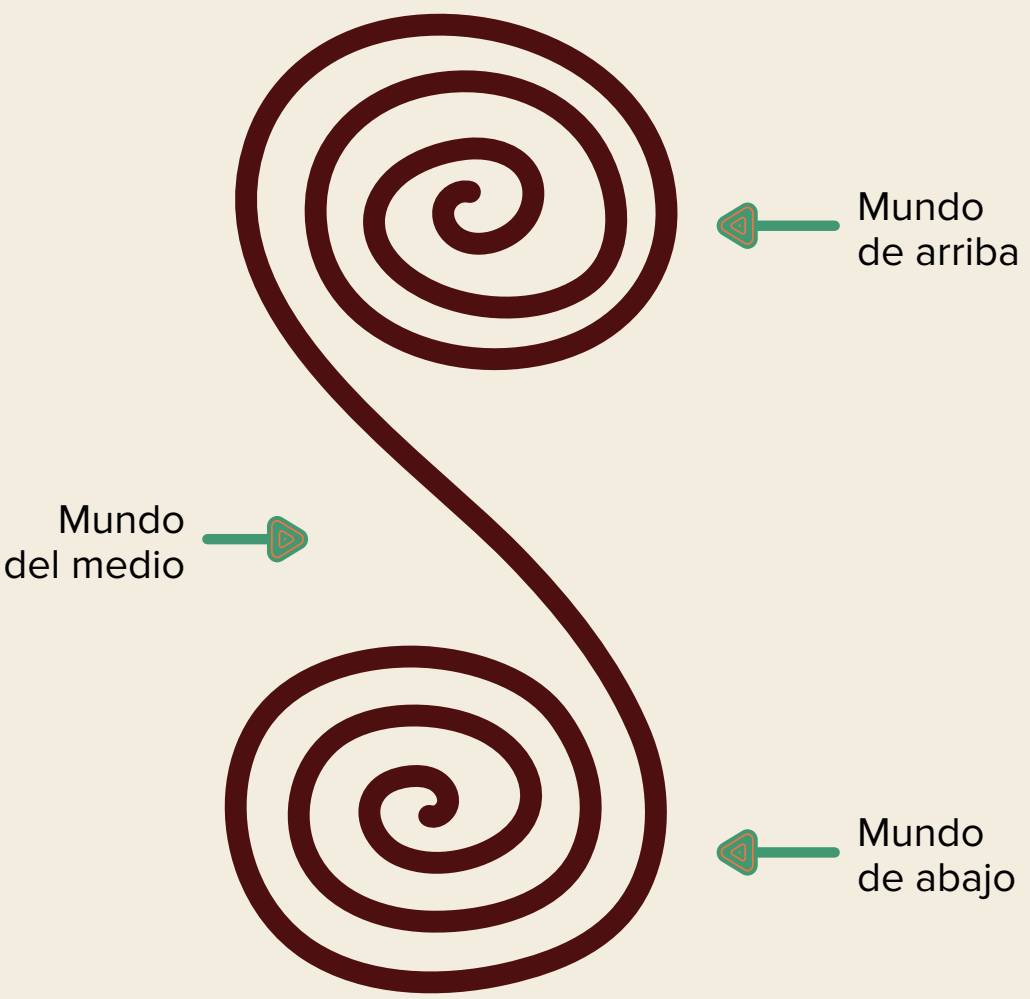




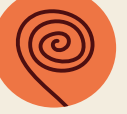

El mundo de arriba es el espacio de las nubes, el cielo, el cosmos de los espíritus mayores, de los dioses de las dimensiones. Representa las cumbres altas, nevadas y frías de los cerros y volcanes, de las extensas zonas de páramo; es tenido como el espacio de los vientos, de las nubes, de los lugares del amanecer, del poder, de la fuerza.

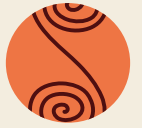

El mundo del medio es el espacio del aquí, en el que andamos y nos desplazamos, donde actuamos y desarrollamos actividades individuales y colectivas. El mundo del medio está en los lugares de clima medio, en las terrazas que se consideran lugares del equilibrio entre lo frío y lo cálido.

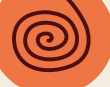

En el mundo de abajo o mundo de adentro se encuentran espacios internos de la tierra, lugares sagrados de entierro de los ancestros, lugar de fuego, energías y organismos. Se representa en los profundos cañones y valles interandinos, en las zonas bajas, cálidas como los huaicos y húmedas de los piedemontes costero y amazónico, son concebidos como lugares del atardecer, de la sabiduría, de la calma.

En el conocimiento científico los objetos de estudio son materiales. El mundo de arriba, es decir el cosmos, es estudiado a través de la cosmología. El mundo del medio es la superficie que habitamos y es estudiado por diferentes ciencias naturales como la física, la química, la biología y la geología, que es una de las ciencias que se ocupan del conocimiento del entorno físico. El mundo de abajo o de adentro, corresponde al subsuelo y es estudiado a partir de investigaciones geológicas, geoquímicas y geofísicas, como las realizadas por el Servicio Geológico Colombiano, para conocer y hacer seguimiento a la actividad del volcán Azufral.

En el mundo de abajo, de acuerdo con el conocimiento tradicional, se encuentra un lugar de fuego y energías, que para el conocimiento científico corresponde con el subsuelo que alberga el sistema magmático (roca fundida caliente), y el sistema geotérmico hidrotermal (agua caliente). Definitivamente fuego y energías. 


\section{El sistema geotérmico hidrotermal del volcán Azufral}

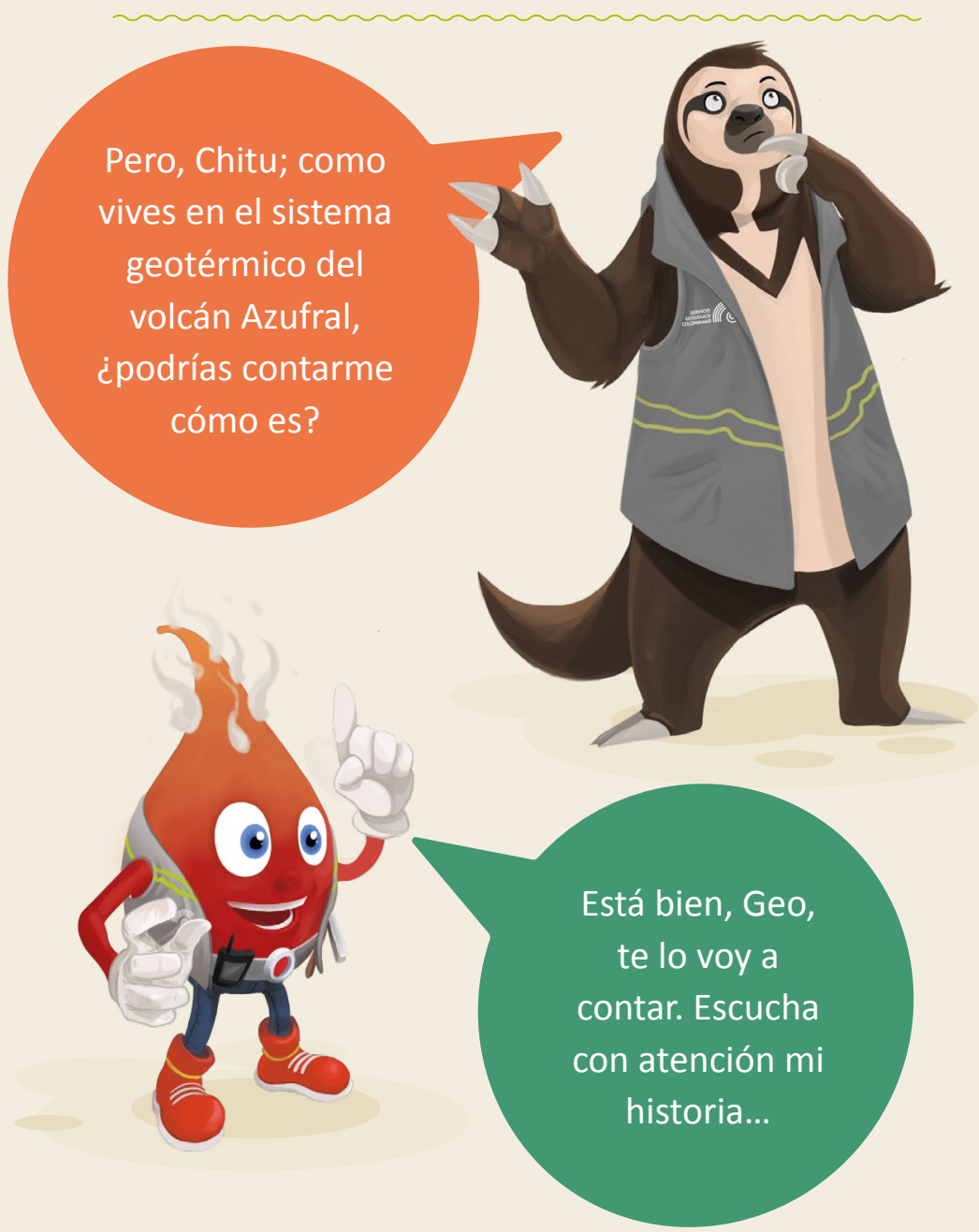

Vivo bajo la superficie de la montaña Gran Chaitán, también llamada volcán Azufral, en un sitio maravilloso y muy caliente, acompañada de muchas otras gotas de agua y de algunas burbujas muy necias y divertidas, a las que llamamos gases. Ellos tienen nombres diferentes y raros como gas carbónico y sulfuro de hidrógeno. Afortunadamente, el sulfuro de hi- 
drogeno es más escaso porque no le gusta bañarse y tiene muy mal olor. Nuestro hogar es muy hermoso y variado; queda como a $2 \mathrm{~km}$ de profundidad y está hecho de rocas calientes con muchos canales y poros, a través de los cuales hacemos paseos muy largos y entretenidos.

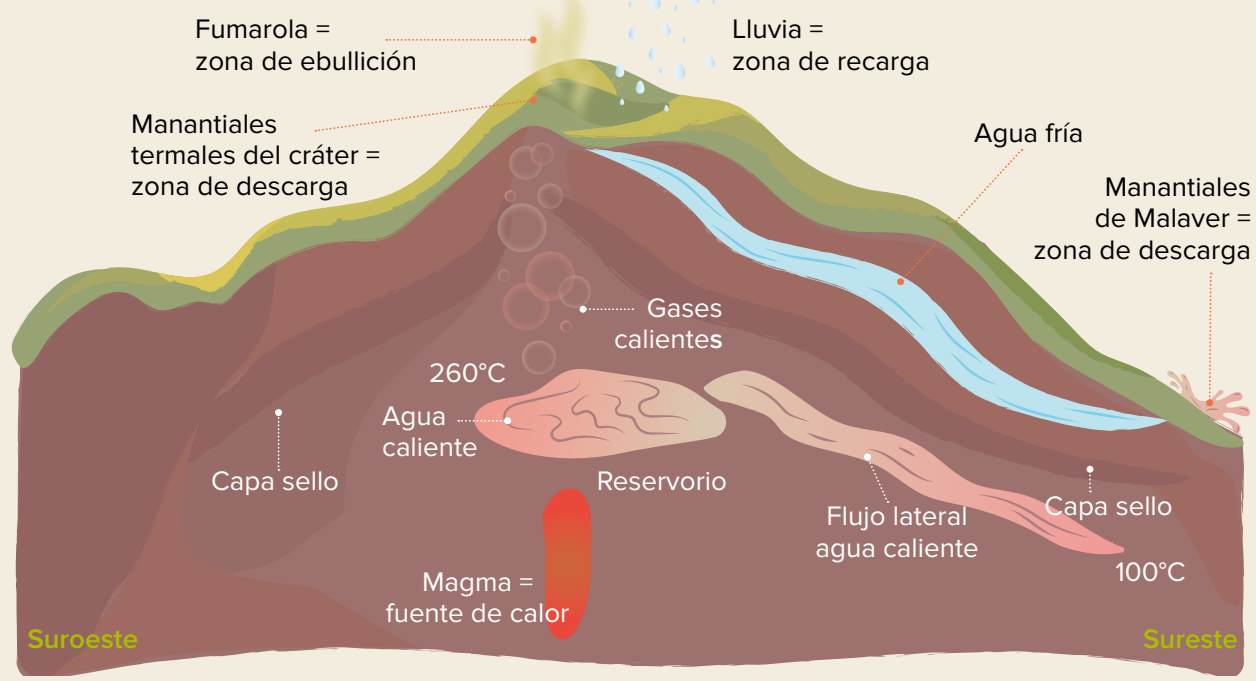

Después de un rato en casa, que algunos llaman reservorio o acuífero, cuando ya estamos calientes, nos volvemos más livianas que las gotas más frías recién llegadas y podemos flotar, encima de ellas, en dirección hacia el techo de casa. El techo, conocido como capa sello, que está muy bien construido en arcilla, nos protege de perder el calor que tanto nos gusta y nos impide salir de casa.

La mayoría de nosotras venimos desde la fría superficie, donde caímos con las lluvias en algunas áreas de la zona alta del Gran Chaitán, llamadas zona de recarga (de agua); después descendimos recorriendo caminos lar- 
gos y estrechos, penetrando suelo y rocas. Pero hay otras gotas que vienen de sitios más profundos y calientes; ellas nos han contado que el calor allá abajo es tan fuerte, que las rocas se vuelven líquidas y calientan otras rocas que se encuentran a su alrededor. El sitio donde se alojan las rocas líquidas se denomina cámara magmática del volcán Azufral; este es el foco de calor de las aguas termales del Gran Chaitán, también llamado fuente de calor.

El techo de casa tiene algunas goteras por donde nos escapamos con gases, para jugar y refrescarnos cerca de la superficie. A través de estas goteras, casi siempre verticales, viajamos juntas a toda velocidad; esta zona de carreras es llamada flujo ascendente. A veces, gases y algunas de nosotras convertidas en vapor, se separan en burbujas formando el lote de punta, que también es conocido como zona de ebullición. Cuando alcanzamos a salir a la superficie sin encontrarnos con acuíferos, a través de rocas rotas nos pueden ver como una descarga de vapor y nos perciben con el olfato, gracias a nuestro amigo, el sulfuro de hidrógeno. Esto es lo que se conoce como fumarola, que en el caso del Gran Chaitán, corresponde con la fumarola de la Laguna Verde. Esta carrera es muy emocionante, pero no siempre termina tan rápido en la superficie. Si nos encontramos con agua fría, algunos metros por debajo de la superficie, nos detenemos a refrescarnos y ahí nos quedamos un tiempito, hasta que la calentamos un poco. Luego, salimos a la superficie por otros canales que llevan agua, que se denominan manantiales termales, y son los que vemos en el Gran Chaitán dentro del cráter. 
En ocasiones nos vamos en grupos grandes, siguiendo unas rutas más largas, de varios kilómetros, escondidas bajo la superficie y protegidas por enramadas o techos de arcilla, que se fueron formando a nuestro paso con los años. Las rutas de este viaje lejos de casa son conocidas como flujo lateral. En el recorrido nos mezclamos con aguas que son muy ricas por su frescura y sabor saladito. Finalmente, solo algunas de nosotras alcanzamos a ver la luz del sol, cuando salimos a la superficie a través de otros canales de agua, como en los manantiales termales de Tercán, Chimangual, Malaver, La Calera y San Ramón. Los sitios en donde hay fumarolas y manantiales son conocidos como zonas de descarga.

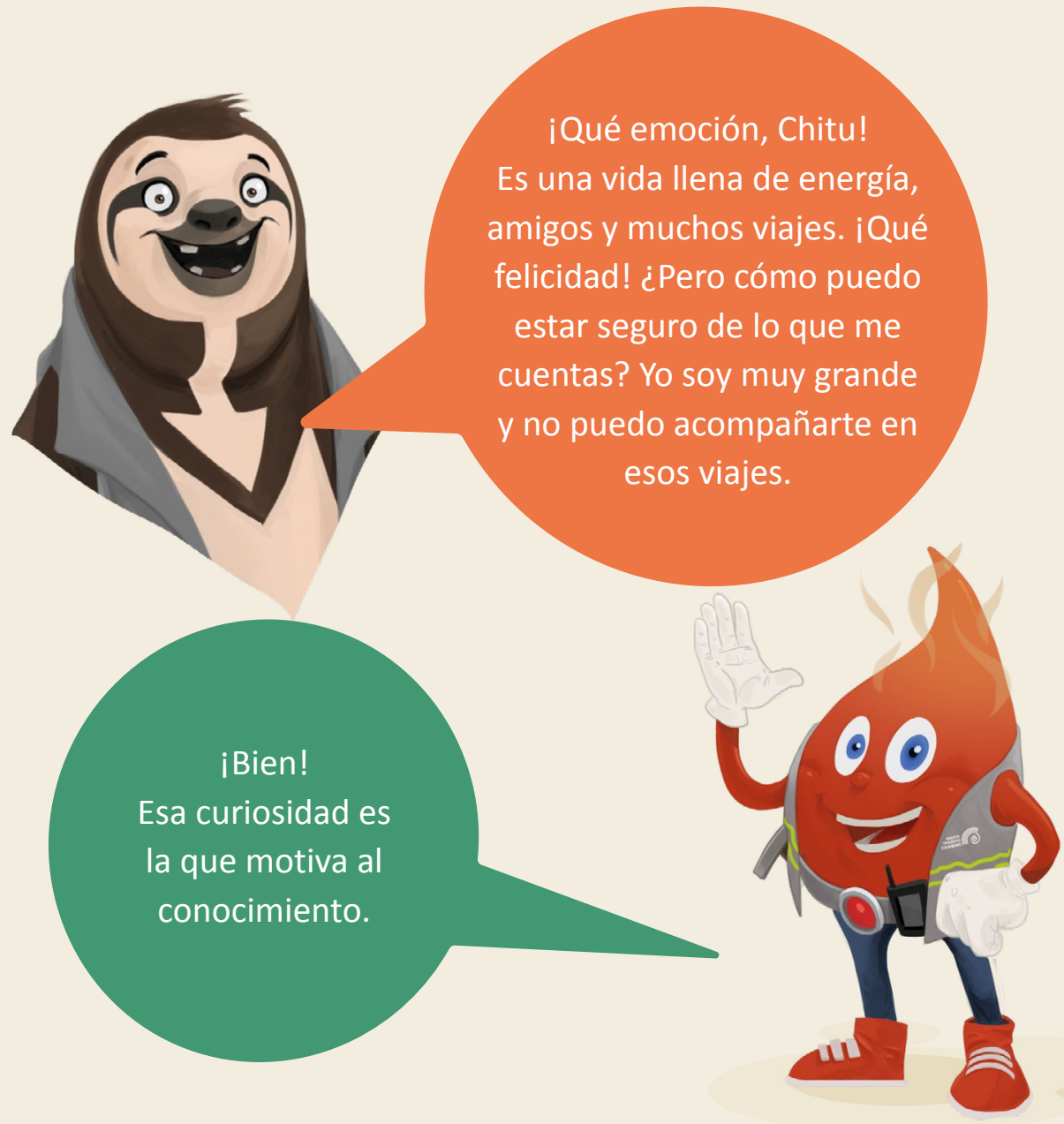


La manera de confirmarlo es observando directamente bajo el suelo. Para poder hacerlo, es necesario excavar agujeros en la tierra. Los agujeros más sencillos son de poca profundidad (trescientos a quinientos metros), se utilizan para sacar y analizar muestras de las rocas y de agua, si se encuentra; también para medir propiedades físicas en las paredes del agujero, especialmente la variación de la temperatura con la profundidad; por eso se llaman agujeros de gradiente térmico.

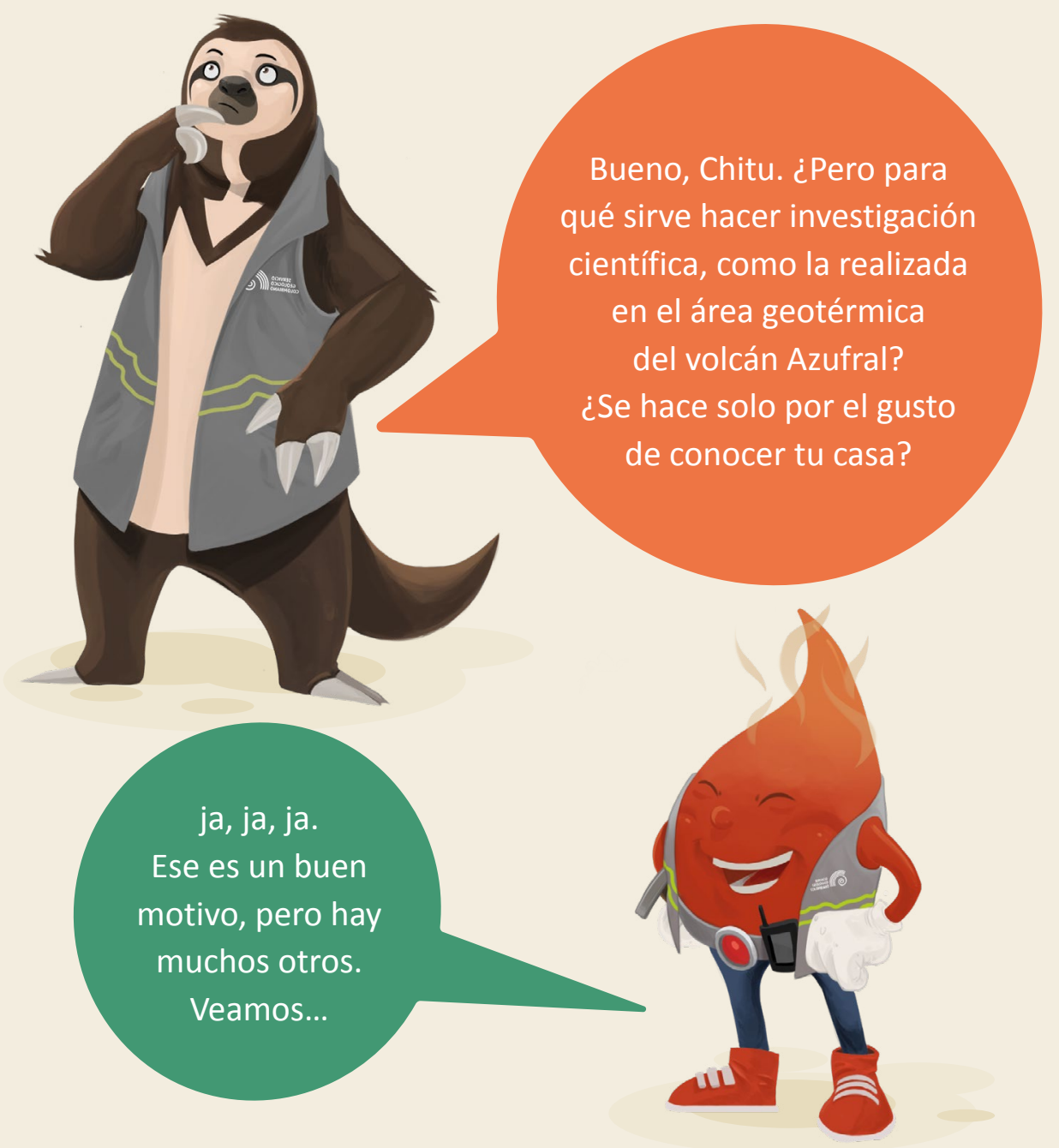




\section{(0)}

"La ciencia misma es el poder", dijo el científico británico Francis Bacon, y "El conocimiento es poder", afirmó el pensador inglés Thomas Hobbes. Son frases que nos recuerdan la posibilidad de que la información y el conocimiento (o acumulación de saberes), le den a la raza humana la capacidad de ser influyentes en los cambios del entorno y de sí mismos. En el caso de las investigaciones que el Servicio Geológico Colombiano realiza en las áreas geotérmicas, la información y el conocimiento dan la posibilidad de:

Responder a preguntas sobre las características del recurso termal, como:

- ¿En dónde está ubicado? ¿A qué profundidad? ¿En qué tipo de rocas?

(4) ¿Qué dimensiones tiene? ¿Cuál es su estructura bajo la superficie?

- ¿Qué edad y composición tienen las rocas que sirven como fuente de calor?

- ¿Qué temperatura tiene el fluido caliente? ¿Cómo es su composición?

- ¿En dónde precipita el agua de lluvia que le sirve de recarga de agua al sistema? ¿Cómo es la circulación o tránsito del agua caliente bajo el subsuelo?

Aportar información de interés para los planes de ordenamiento de los territorios: Identificar zonas que requieren de protección, como las de recarga de agua; identificar oportunidades y restricciones que ofrece el recurso termal, contribuir con información para evaluar estabilidad del terreno.

Ampliar el conocimiento que tenemos del volcán, cuyo interior alberga no solo al sistema magmático (rocas fundidas calientes), sino también un sistema de agua caliente (geotérmico), con el que coexiste. 
(D) Identificar riquezas ancestrales de las comunidades indígenas locales, enterradas en el subsuelo, así como posibles fuentes de suministro de agua, principalmente si se llevan a cabo excavaciones o perforaciones.

(D) Conocer el tipo de agua de los manantiales termales y establecer su vocación para el uso terapéutico.

(D) Apropiarse de un recurso nativo y conocerlo para tomar decisiones autónomas, independientes e informadas sobre el mismo.

\section{Manantiales y fumarola del sistema hidrotermal del volcán Azufral}

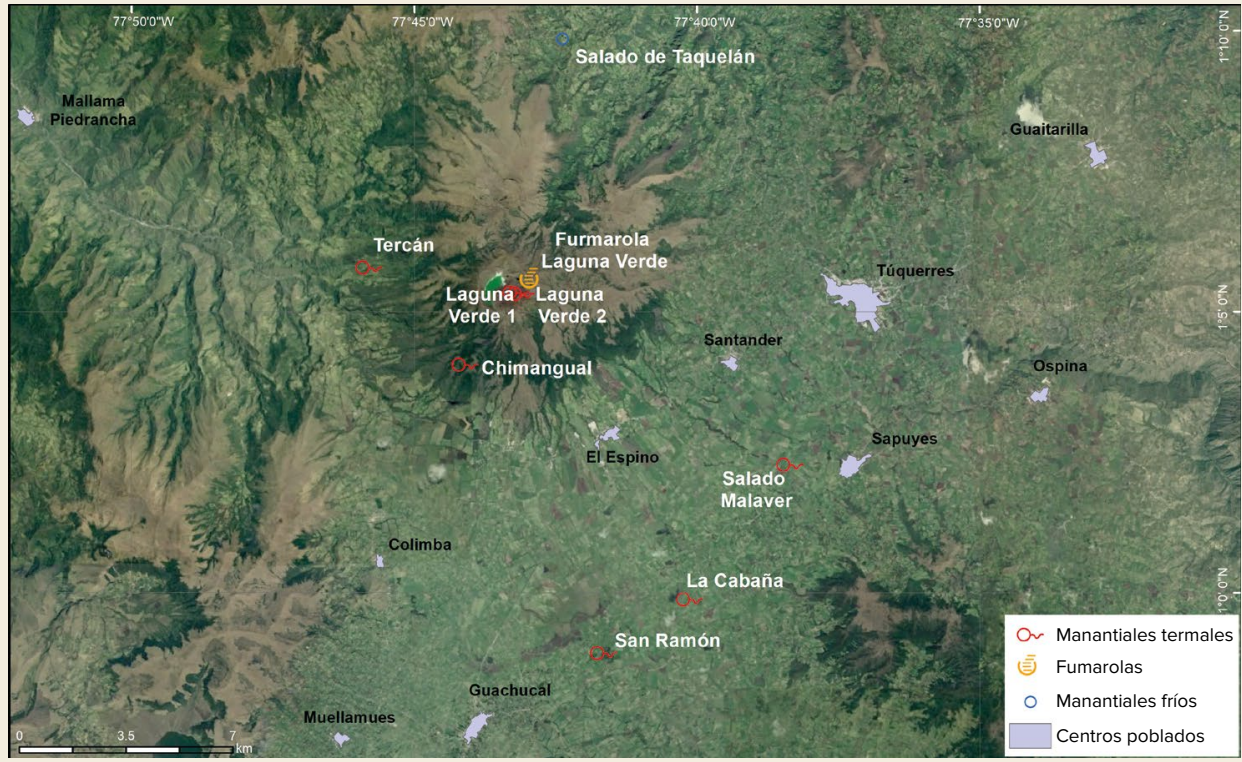

Imagen de satélite que muestra las manifestaciones termales en el área geotérmica del volcán azufral

Fuente: Google Earth. 


\section{Salado de Tequelán}

Temperatura: $14^{\circ} \mathrm{C}$

pH: 6,6

Conductividad: $13220 \mu \mathrm{S} / \mathrm{cm}$

Composición: bicarbonatada sodica

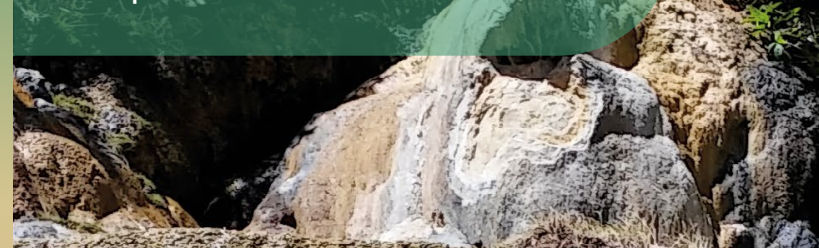

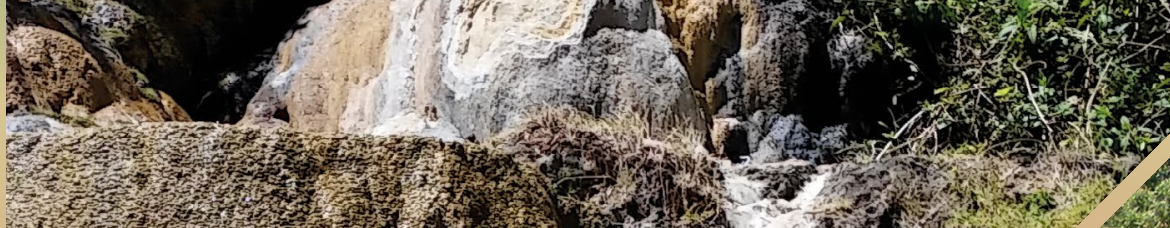
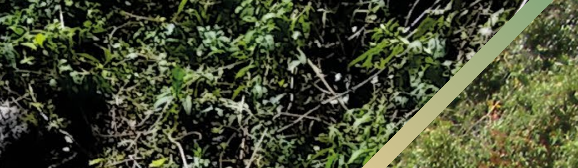

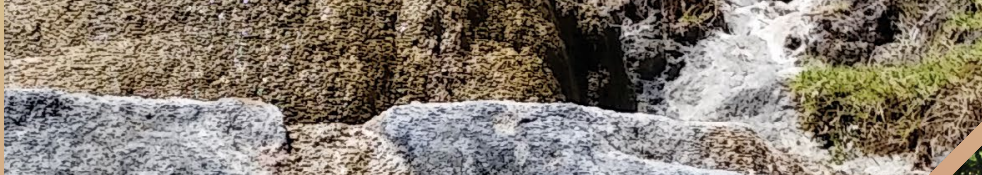

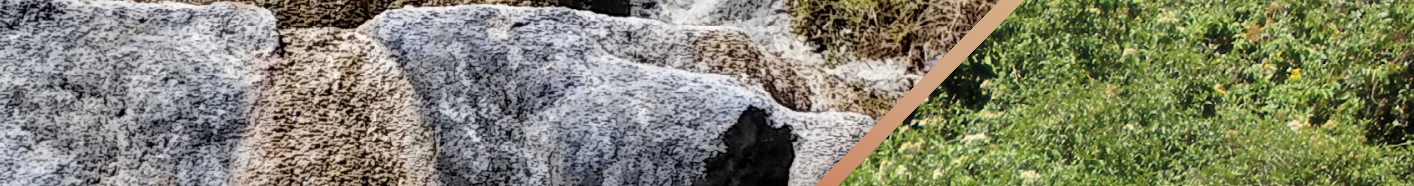

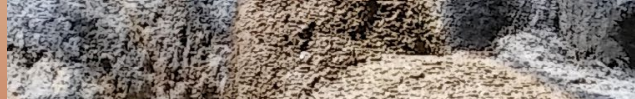

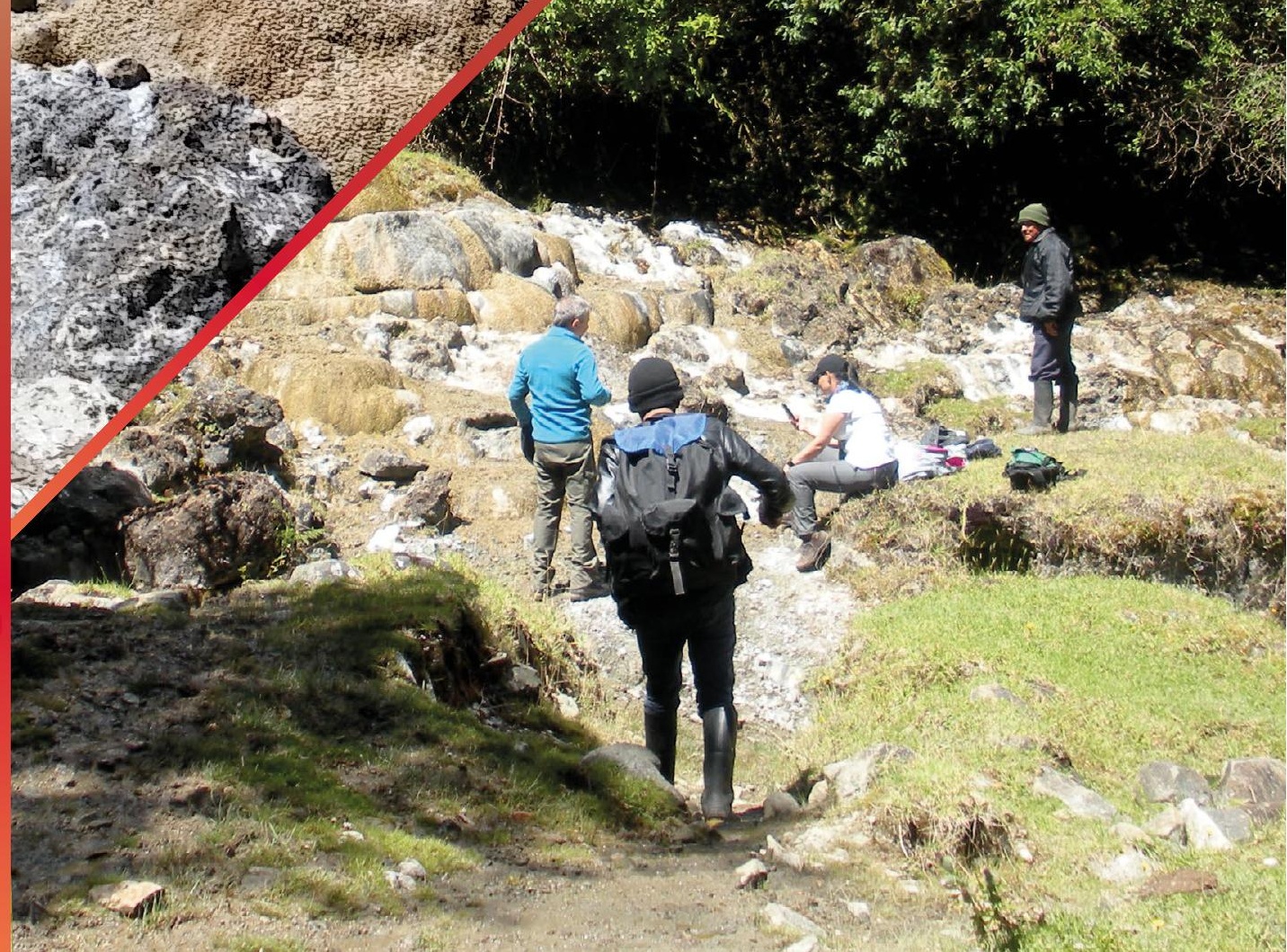




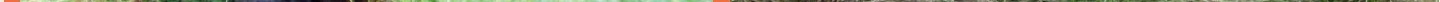




\section{Ahora sí, a divertirnos con lo aprendido}

Resuelve el crucigrama que contiene muchos de los términos relacionados con los estudios científicos, que realiza el Servicio Geológico Colombiano en el volcán Azufral. La solución al final.

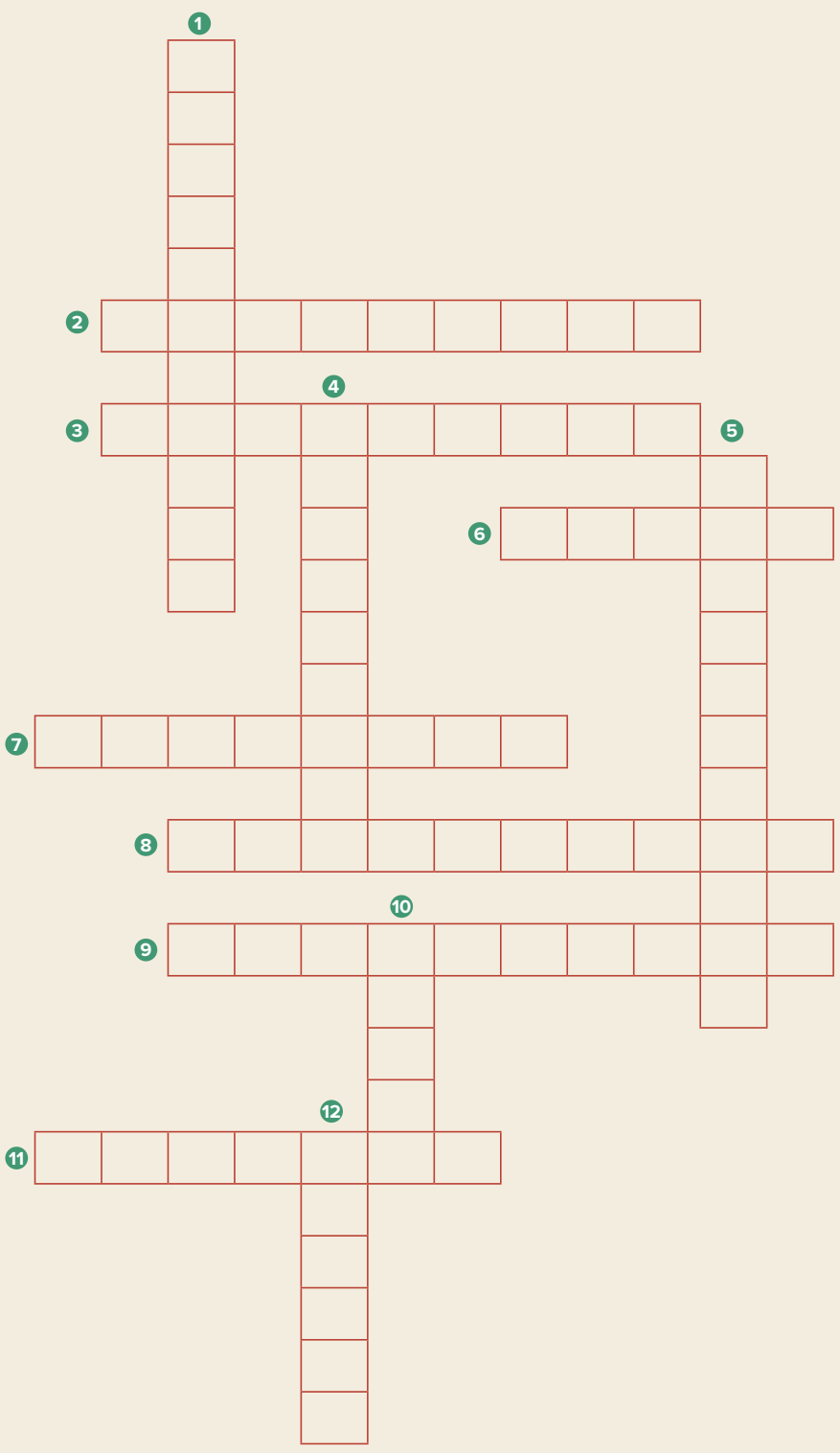




\section{Verticales}

1. Es el nombre que recibe el lago que se encuentra dentro del cráter del Gran Chaitán.

4. Municipio colombiano que se encuentra localizado a $11 \mathrm{~km}$ al oriente del Gran Chaitán.

5. Es el nombre que recibe el volcán Azufral de acuerdo con la cosmogonía de las comunidades indígenas.

10. Se refiere a la roca fundida.

12. Animal que se encuentra en los páramos y zona boscosa del Gran Chaitán.

\section{Horizontales}

2. Es la expresión superficial más típica de los sistemas geotérmicos hidrotermales.

3. Es el nombre que se le da al calor que se encuentra al interior de la Tierra.

6. Palabra de origen Quechua que traduce: "Espiral del Universo ordenado".

7. Es una de las expresiones en el subsuelo en el cual se encuentran alojadas las aguas calientes.

8. Es un espacio del subsuelo donde se alojan las aguas calientes.

9. Es un manantial hermoso por tener una cascada de agua caliente.

11. Personaje principal en la cosmogonía del volcán Azufral que luchó contra el Guangas. 


\section{Respuestas al crucigrama}

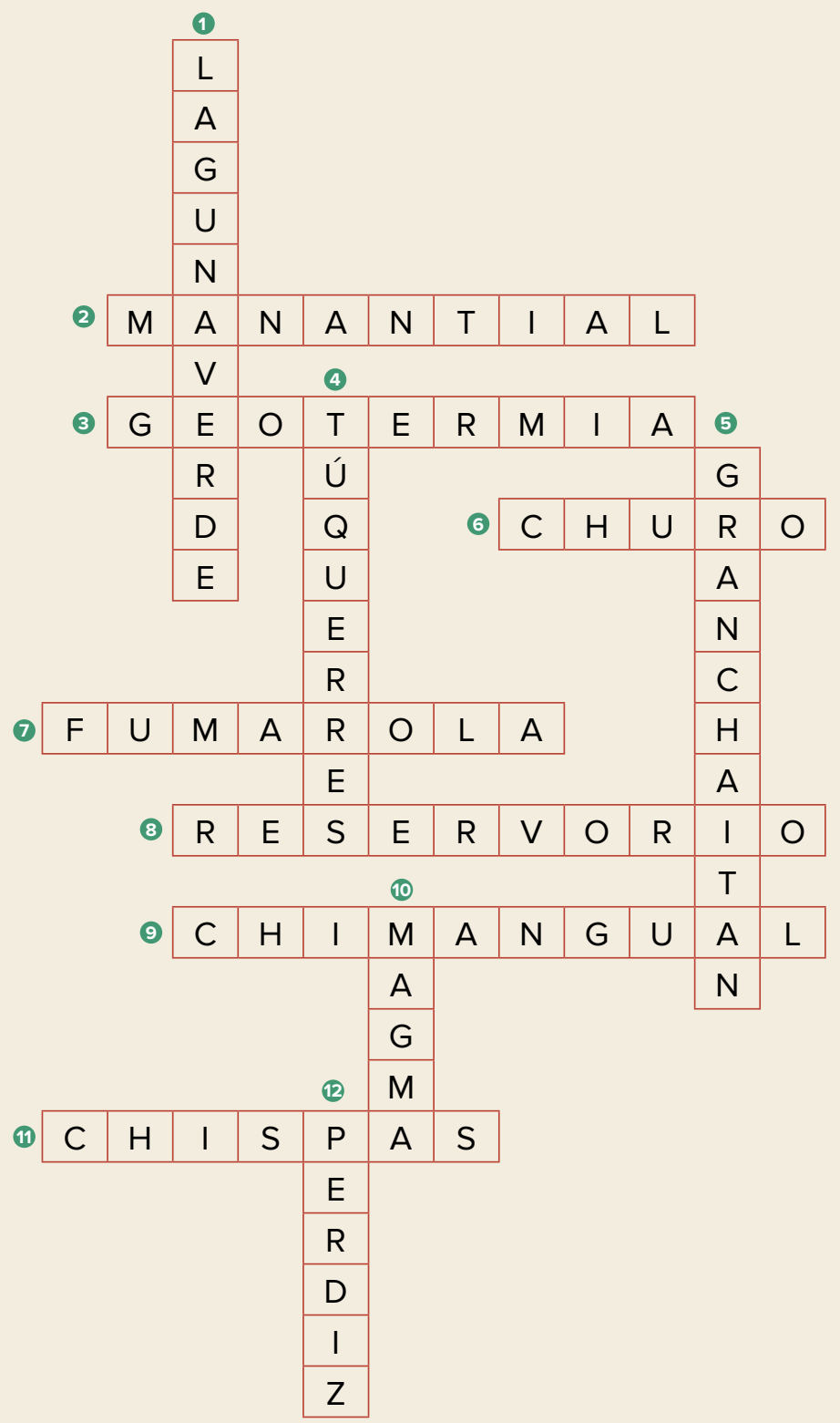


Encuentra en la sopa de letras las palabras relacionadas con los estudios científicos que realiza el Servicio Geológico Colombiano en el volcán Azufral.

\begin{tabular}{|c|c|c|c|c|c|c|c|c|c|c|c|c|c|}
\hline A & L & F & S & O & X & P & D & T & I & C & D & I & U \\
\hline X & D & G & E & O & T & E & R & M & I & A & H & S & F \\
\hline M & G & R & A & N & C & H & A & I & T & A & N & P & Z \\
\hline E & U & U & M & N & K & Z & D & O & Z & J & R & U & D \\
\hline B & U & P & H & S & A & Z & U & F & R & A & L & D & D \\
\hline R & D & E & D & O & D & E & D & I & O & S & R & H & T \\
\hline P & E & Q & P & E & R & D & I & C & E & S & Q & N & I \\
\hline T & U & K & O & V & T & K & X & C & H & I & T & U & K \\
\hline C & H & I & S & P & A & S & D & W & W & Y & E & R & R \\
\hline P & E & T & R & O & G & L & I & F & O & M & J & W & I \\
\hline R & G & X & P & U & S & A & P & U & Y & E & S & N & W \\
\hline J & F & U & M & A & R & O & L & A & Y & I & C & C & A \\
\hline D & V & L & A & G & U & N & A & V & E & R & D & E & H \\
\hline M & A & N & A & N & T & I & A & L & L & G & M & I & T \\
\hline
\end{tabular}

Azufral / Chitu / Fumarola / Gran Chaitán / Manantial / Petroglifo / Chispas / Dedo de Dios / Geotermia / Laguna Verde / Perdices / Sapuyes 


\section{Referencias}

Aza Cuaical, D. M. (2016). Iconografía Pastos un saber ancestral (tesis de grado). Universidad del Valle.

Fundación de Estudios e Investigaciones Sociopolíticas, Económicas y Culturales. Fundación Proyectar. (2009.) Manual de justicia del territorio indígena de Túquerres. Disponible en https://siic.mininterior.gov.co/ sites/default/files/upload/SIIC/Reglamentos/nternos/mj_tuquerres. pdf

Servicio Geológico Colombiano (SGC). (2015). Inventario nacional de manifestaciones hidrotermales. Aplicativo web en http://hidrotermales. sgc.gov.co/

Patiño, J., Ponce, M., Oviedo, O., Tatamues, E., Mayag, I. y Tello, E. (2009). Manual de justicia propia del territorio indígena de Túquerres y Reglamento interno y de convivencia del pueblo indígena del territorio de Túquerres. Universidad Internacional de la Florida y Gobernación del departamento de Nariño. 

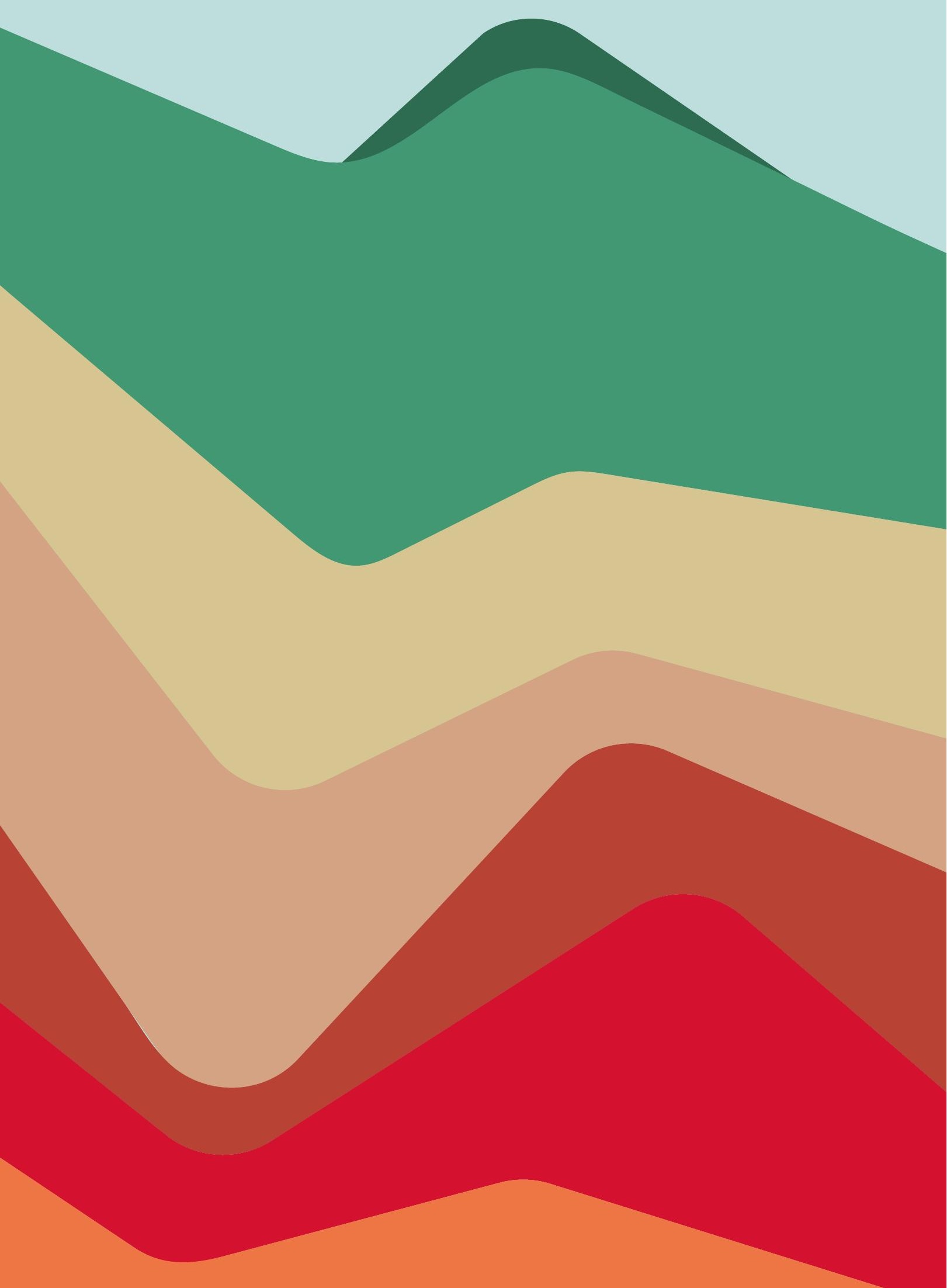
